\title{
Extraction and Filter Algorithm of Guidance Information for Full- strapdown Seeker on Rotation Missile
}

\author{
YongShan Liu ${ }^{1}$, Li Song ${ }^{1}$, and JingLong Li ${ }^{1}$ \\ ${ }^{1}$ School of Aerospace Engineering, Beijing Institute of Technology, China
}

\begin{abstract}
Strapdown seekers are superior to platform seekers for their simple structure, high reliability and light weight but cannot measure the line-of-sight angle rate information for the guidance of rotation missile directly. This paper aims at the engineering application of full-strapdown seekers on rotation missile problem. Firstly, a line-of-sight angle rate solution model is established. Based on the MATLAB, the extended Kalman filter (EKF) algorithm and unscented Kalman filter (UKF) algorithm are used to estimate the line-of-sight angle rate information of the full-strapdown seekers. The results show that using EKF filter and UKF filter both can obtain effective guidance information and the UKF's effect is better.
\end{abstract}

\section{INTRODUCTION}

Unlike traditional platform seeker, the full-strapdown seeker attaches all hardware to the projectile, removes the stable platform and the control mechanism. Which has the important significance on reducing the cost of seeker and improving the system reliability. Whereas the fullstrapdown seeker cannot directly obtain the line-of-sight rate by its physical tracking loop and need digital calculation methods. Rotary missile system has the advantages of smaller size, lower cost, less control equipment, and can reduce the interference caused by the asymmetry of the projectile. However, the spin of the missile body will cause cross coupling between the pitch and yaw channels. Creagh MA et al. got the results through simulating which shows when the rotational speed of the projectile is high, the coupling caused by rolling becomes more serious, and due to the spin of the rotating projectile, the pitching channel and yawing channel of the rotating projectile will be cross-coupled [1].

As mentioned previously, the other estimation method of inertial line-of-sight rate is designed based on relative motion between missile and target, where many estimation algorithms are proposed according to the different filters adopted. Ehrich et al. put the additional rate compensation + differential network method to build single channel line-of-sight angle and then enter the guidance system through a low-pass filter [2]. Kim et al. [3] put forward a method to derive such correlation and finally obtained the inertial line-of-sight rate. During the deriving process, the body line-of-sight rate was calculated by a differential network. Bai Rui et al. [4] based on the typical launch conditions of small air-tosurface missile established an estimation algorithm and used the cubature Kalman filter for the rate estimation. Sun et al. [5] estimated the inertial line-of-sight rate of strapdown optical seeker using unscented Kalman filter (UKF) method, consider the estimation accuracy mainly depends on the body line-of-sight angle accuracy and gyro accuracy and simulate these two factors. Waldmann $\mathbf{J}[6]$ describes the modeling of an imaging seeker and the formulation of an extended Kalman filter for the estimation of line-of-sight rate from measurements of relative angular displacement between seeker gimbals and a low-cost strapdwn inertial unit. Li, J. J. [7] used the pursuit guidance law and proportion guidance law in order to get the line-of-sight rate and through amending "innovation" in the equations of Kalman filter, outliereliminating Kalman filtering algorithm is achieved.

In this paper, first based on the information coupling problem of rotation missile and the full-strapdown seeker problem, a decoupled model is established to extract the inertial the line-of-sight angle rate information from the observation data of full-strapdown seekers. And then by using the extended Kalman filter (EKF) algorithm and the unscented Kalman filter (UKF) algorithm, the real-time estimation of projectile guidance information is simulated and last verified the effectiveness of the algorithms.

\section{LINE-OF-SIGHT ANGLE RATE MODEL}

In this section, define four coordinate systems and the transformation between each coordinate system at the outset. Through introducing the missile-target distance and the approaching speed, the standard state equations and observation equations are presented. Getting the relation between the state equations and observation equation, the inertial line-of-sight elevation angle rate and the inertial line-of-sight azimuth angle rate are obtained. 


\subsection{Coordinate system definition}

The following coordinate systems and angles will be used in this paper[8]:the inertial coordinate system $O_{e} x_{e} y_{e} z_{e}$, the body coordinate system $O x_{b} y_{b} z_{b}$, the line-of-sight coordinate system $O x_{s} y_{s} z_{s}$, and the body line-of-sight coordinate system $O x_{l} y_{l} z_{l}$. The inertial lineof-sight elevation angle $q_{\gamma}$, the inertial line-of-sight azimuth angle $q_{\lambda}$,the body line-of-sight elevation angle $q_{\alpha}$, the body line-of-sight azimuth angle $q_{\beta}$, the pitch angle $\theta$, the yaw angle $\psi$, the roll angle $\gamma$. The conversion of each coordinate system is shown in Fig.1.

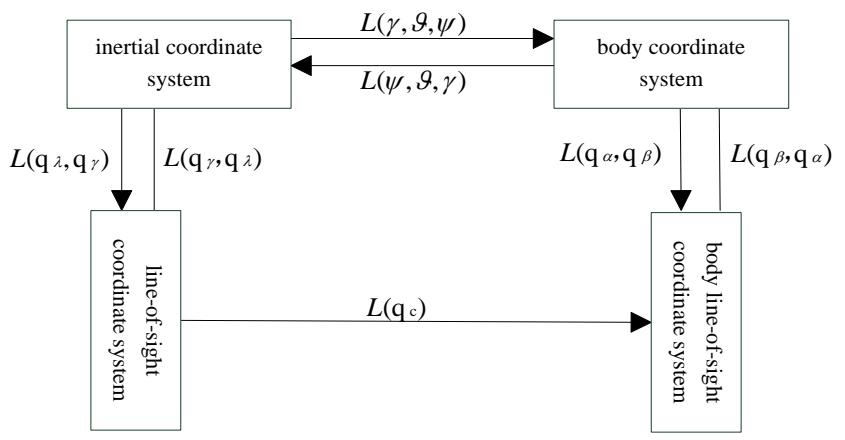

Fig 1. Conversion of each coordinate system.

\subsection{Line-of-sight Information state equations}

According to the kinematics and geometric relations of the rotation missile and target in space[9-10], the rotational angular velocity of the line-of-sight coordinate system $O x_{s} y_{s} z_{s}$ relative to the ground coordinate system $O_{e} x_{e} y_{e} z_{e}$ is:

$$
\omega_{s}=\dot{q}_{\lambda} \sin q_{\gamma} \dot{i}_{s}+\dot{q}_{\lambda} \cos q_{\gamma} \dot{j}_{s}+\dot{q}_{\gamma} \dot{k}_{s}
$$

$\dot{r}$ in Eq.(2) represents the change rate of the missiletarget distant ,and in the line-of-sight coordinate system $O x_{s} y_{s} z_{s}$ has the following relationship:

$$
\begin{aligned}
& \dot{r}_{x}=r \dot{q}_{\lambda} j-r \dot{q}_{\lambda} \cos q_{\gamma} k \\
& \dot{r}_{y}=r \dot{q}_{\lambda} \sin q_{\gamma} k-r \dot{q}_{\lambda} i \\
& \dot{r}_{z}=r \dot{q}_{\lambda} \cos q_{\gamma} i-r \dot{q}_{\lambda} \sin q_{\gamma} j
\end{aligned}
$$

Differentiate the missile-target relative speed $V$ to obtain the missile-target relative acceleration $a$ is:

$$
a=\dot{V}=\left[\begin{array}{c}
\ddot{r}-r \dot{q}_{\gamma}^{2}-r \dot{q}_{\lambda}^{2} \cos ^{2} q_{\gamma} \\
r \ddot{q}_{\gamma}+2 V \dot{q}_{\gamma}+r \dot{q}_{\lambda}^{2} \sin q_{\gamma} \cos q_{\gamma} \\
2 r \dot{q}_{\lambda} \dot{q}_{\gamma} \sin q_{\gamma}-2 V \dot{q}_{\lambda} \cos q_{\gamma}-r \ddot{q}_{\lambda} \cos q_{\gamma}
\end{array}\right]
$$

Assume that the target does not motorize, that is to say the missile-target relative acceleration $a=0$, the Eq. (3) can be obtained:

$$
\left\{\begin{array}{l}
\ddot{r}=r\left(\dot{q}_{\gamma}^{2}+\dot{q}_{\lambda}^{2} \cos ^{2} q_{\gamma}\right) \\
\ddot{q}_{\gamma}=\frac{-2 V \dot{q}_{\gamma}}{r}-\dot{q}_{\lambda}^{2} \sin q_{\gamma} \cos q_{\gamma} \\
\ddot{q}_{\lambda}=2 \dot{q}_{\lambda} \dot{q}_{\gamma} \tan q_{\gamma}-\frac{2 V \dot{q}_{\lambda}}{r}
\end{array}\right.
$$

The state vector of the system can be augmented as $x=\left[\begin{array}{llll}x_{1} & x_{2} & x_{3} & x_{4}\end{array}\right]^{T}=\left[\begin{array}{llll}q_{\lambda} & \dot{q}_{\lambda} & q_{\gamma} & \dot{q}_{\gamma}\end{array}\right]^{T}$.Thus, the line-of-sight information state equations are derived by combining Eq. (4) as follows:

$$
\left\{\begin{array}{l}
\dot{x}_{1}=x_{2} \\
\dot{x}_{2}=-\frac{2 V}{r} x_{2}+2 x_{2} x_{4} \tan x_{3} \\
\dot{x}_{3}=x_{4} \\
\dot{x}_{4}=-x_{2}^{2} \sin x_{3} \cos x_{3}-\frac{2 V}{r} x_{4}
\end{array}\right.
$$

\subsection{Observation Equations}

Assume the position of the target in the line-of-sight coordinate system and the body line-of-sight coordinate system are both $\left[\begin{array}{lll}R & 0 & 0\end{array}\right]$. And the coordinates of the target and the missile in the inertial coordinate system are $\left[\begin{array}{lll}x_{e T} & y_{e T} & z_{e T}\end{array}\right]^{T}$ and $\left[\begin{array}{lll}x_{e D} & y_{e D} & z_{e D}\end{array}\right]^{T}$. According to the conversion relationship between the line-of-sight coordinate system $O x_{s} y_{s} z_{s}$ and the inertial coordinate system $O_{e x e y e z e}$, the relative equations can be written as:

$$
\left[\begin{array}{l}
x_{T D} \\
y_{T D} \\
z_{T D}
\end{array}\right]=\left[\begin{array}{l}
x_{e T} \\
y_{e T} \\
z_{e T}
\end{array}\right]-\left[\begin{array}{l}
x_{e D} \\
y_{e D} \\
z_{e D}
\end{array}\right]=\left[\begin{array}{c}
R \cos q_{\gamma} \cos q_{\lambda} \\
R \sin q_{\gamma} \\
-R \cos q_{\gamma} \sin q_{\lambda}
\end{array}\right]
$$

The geometric relative equations are Eq. (7).

$$
\left\{\begin{array}{l}
q_{\gamma}=\arctan \left(\frac{y_{T D}}{\sqrt{x_{T D}^{2}+z_{T D}^{2}}}\right) \\
q_{\lambda}=\arctan \left(-\frac{z_{T D}}{x_{T D}}\right)
\end{array}\right.
$$

In the similar way, define the coordinates of the target and missile in the body coordinate system are $\left[\begin{array}{lll}x_{b T} & y_{b T} & z_{b T}\end{array}\right]^{T}$ and $\left[\begin{array}{lll}x_{b D} & y_{b D} & z_{b D}\end{array}\right]^{T}$. The conversion relationship between the body coordinate system $O x_{b} y_{b} z_{b}$ and the body line-of-sight coordinate system $O x_{l} y_{l} z_{l}$ : 


$$
\left[\begin{array}{c}
\tilde{x}_{T D} \\
\tilde{y}_{T D} \\
\tilde{z}_{T D}
\end{array}\right]=\left[\begin{array}{c}
x_{b T} \\
y_{b T} \\
z_{b T}
\end{array}\right]-\left[\begin{array}{l}
x_{b D} \\
y_{b D} \\
z_{b D}
\end{array}\right]=\left[\begin{array}{c}
R \cos q_{\alpha} \cos q_{\beta} \\
R \sin q_{\alpha} \\
-R \cos q_{\alpha} \sin q_{\beta}
\end{array}\right]
$$

The geometric relations are:

$$
\left\{\begin{array}{l}
q_{\alpha}=\arctan \left(\frac{\tilde{y}_{T D}}{\sqrt{\tilde{x}_{T D}^{2}+\bar{z}_{T D}^{2}}}\right) \\
q_{\beta}=\arctan \left(-\frac{\bar{z}_{T D}}{\tilde{x}_{T D}}\right)
\end{array}\right.
$$

$R_{i j}$ is the element of the conversion matrix $C_{e}^{b}$ in the $i$ th row and $j$ th column which is the coordinate transformation matrix from the ground coordinate system to the body coordinate system,. The conversion $C_{e}^{b}$ matrix:

$$
C_{e}^{b}=\left[\begin{array}{ccc}
\cos \vartheta \cos \psi & \sin \vartheta & -\cos \vartheta \sin \psi \\
-\sin \vartheta \cos \psi \cos \gamma+\sin \psi \sin \gamma & \cos \vartheta \cos \gamma & \sin \vartheta \sin \psi \cos \gamma+\cos \psi \sin \gamma \\
\sin \vartheta \cos \psi \sin \gamma+\sin \psi \cos \gamma & -\cos \vartheta \sin \gamma & -\sin \vartheta \sin \psi \sin \gamma+\cos \psi \cos \gamma
\end{array}\right]
$$

Combine the Eq. (6), Eq. (8) and Eq. (10) can get:

$$
\left[\begin{array}{c}
R \cos q_{\alpha} \cos q_{\beta} \\
R \sin q_{\alpha} \\
-R \cos q_{\alpha} \sin q_{\beta}
\end{array}\right]=\left[\begin{array}{lll}
R_{11} & R_{12} & R_{13} \\
R_{21} & R_{22} & R_{23} \\
R_{31} & R_{32} & R_{33}
\end{array}\right]\left[\begin{array}{c}
R \cos q_{\gamma} \cos q_{\lambda} \\
R \sin q_{\gamma} \\
-R \cos q_{\gamma} \sin q_{\lambda}
\end{array}\right]
$$

Angle information provided by full-strapdown seeker includes the body line-of-sight angle $q_{\alpha}$ and $q_{\beta}$, which are considered as the observation variables. Under this circumstances, defining the line-of-sight observation vector $y=\left[\begin{array}{ll}q_{\alpha} & q_{\beta}\end{array}\right]^{T}=\left[\begin{array}{ll}y_{1} & y_{2}\end{array}\right]^{T}$. The line-of-sight observation equations of the full-strapdown seeker can be written as:

$\left\{\begin{array}{l}y_{1}=\arcsin \left(R_{21} \cos x_{3} \cos x_{1}+R_{22} \sin x_{3}-R_{23} \cos x_{3} \sin x_{1}\right) \\ y_{2}=-\arctan \left(\frac{R_{31} \cos x_{1}+R_{32} \tan x_{3}-R_{33} \sin x_{1}}{R_{11} \cos x_{1}+R_{12} \tan x_{3}-R_{13} \sin x_{1}}\right)\end{array}\right.$

So far, the line-of-sight angle information state equations and the observation equations of the of the fullstrapdown seeker on the rotating missile are established. From Eq. (5) and Eq. (12), it can be seen that there is strong nonlinearity existing both in the line-of-sight state equations and observation equations.

\section{Rotation Missile Guidance Information Estimation and Simulation}

In this paper, the Extended Kalman Filter (EKF) and Unscented Kalman Filter (UKF) algorithm are simulated and verified under both the navigation system measurement data and the stapling target coordinates have errors. In the inertial coordinate system, the actual position of the target is $(0 \mathrm{~m}, 2.5 \mathrm{~m}, 0 \mathrm{~m})$, the coordinate of stapling target is $(100 \mathrm{~m}, 52.5 \mathrm{~m}, 60 \mathrm{~m})$. That is, the stapling target measurement error is $(100 \mathrm{~m}, 50 \mathrm{~m}, 60 \mathrm{~m})$. The initial position of the missile in the ground coordinate system is $(-7900 \mathrm{~m}, 2050 \mathrm{~m}, 60 \mathrm{~m})$.

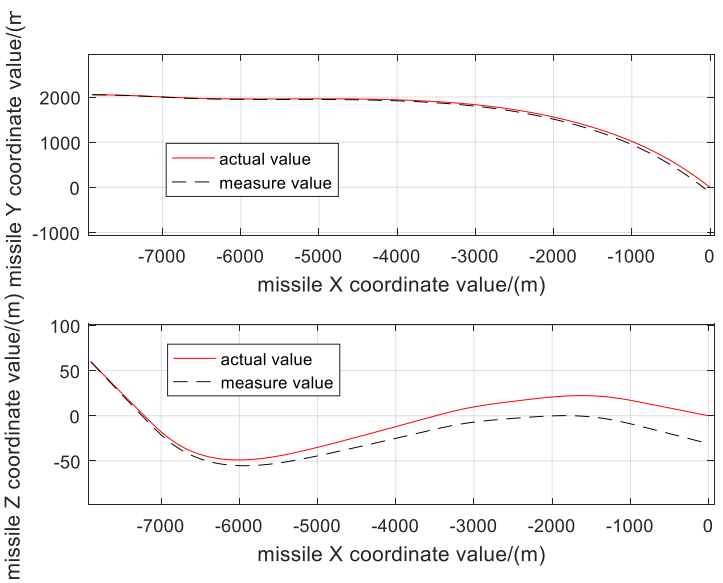

Fig 2. Missile X, Y, Z location.

It can be seen from the Fig. 2 that the measurement errors between the actual position and measurement position of the rotation missile in the inertial navigation system gradually increases with time. Therefore, directly using the data measured by the inertial navigation system to guide would miss targets.

In the process of the rotation missile flying to the target, the body line-of-sight elevation angle $q_{\alpha}$ and the body line-of-sight azimuth angle $q_{\beta}$ are shown in Fig. 3 .


Fig 3. Body line of sight and partial enlargement.

Since the rotating missile will generate the pitch and yaw directions coupling during the movement, the body line-of-sight elevation angle $q_{\alpha}$ and the body line-of-sight azimuth angle $q_{\beta}$ in Fig. 3 are same size and 90 degrees phase difference. Due to the low repetition rate of the laser pulse code, the measured signals of the body lineof-sight elevation angle and the body line-of-sight azimuth angle are not perfect as sine functions. 


\subsection{Estimation of Guidance Information Based on EKF and UKF Algorithms}

The stochastic nonlinear discrete formula for the extended Kalman (EKF) filtering algorithm [11] is:

$$
\begin{gathered}
X_{k}=\Phi_{k, k-1} X_{k-1}+\Gamma\left(\hat{X}_{k-1, k-1}\right) \mathrm{W}_{k-1} \\
Z_{k}=H_{k} X_{k}+V_{k}
\end{gathered}
$$

In the Eq.(13) and Eq.(14), $W$ is the process noise matrix, $V$ is the observation noise matrix.

The line-of-sight information of rotating missile has strong nonlinear characteristics. When applying extended Kalman filter, the Jacobian matrix is needed to obtain the deviation based approximate linear equations of the state equations.

$$
J_{k, k-1}=\frac{\partial f}{\partial X_{k-1}^{*}}=\left.\frac{\partial f\left(\mathrm{X}_{k-1}, \mathrm{k}-1\right)}{\partial X_{k-1}}\right|_{X_{k-1}=X_{k-1}^{n}}
$$

Discretize the Jacobian matrix:

$$
\Phi_{k, k-1}=I+J_{k, k-1} \cdot \Delta \mathrm{t}
$$

Unscented kalman filter (UKF) algorithm firstly needs to designs a series of Sigma points. Then calculating the result of $f(\cdot)$ with the set sigma points, and $(\overline{\mathrm{Z}}, \mathrm{Pz})$ based on $\chi_{i}$.

$$
\left\{\begin{array}{l}
\xi_{k-1}^{(0)}=\hat{X}_{k-1}^{a} \\
\xi_{k-1}^{(\mathrm{i})}=\hat{X}_{k-1}^{a}+\left(\sqrt{(\mathrm{n}+\lambda) \mathrm{P}_{k-1}^{a}}\right)_{i}, i=1,2, \ldots, n \\
\xi_{k-1}^{(\mathrm{i})}=\hat{X}_{k-1}^{a}-\left(\sqrt{(\mathrm{n}+\lambda) \mathrm{P}_{k-1}^{a}}\right)_{i}, i=n+1, \mathrm{n}+2, \ldots, 2 n
\end{array}\right.
$$

Propagate $\chi_{k, k-1}$ through nonlinear observation function $H(\cdot)+r_{k}$. From $\chi_{k, k-1}$ can get the output prediction $\hat{Z}_{k, k-1}$, as well as the self-covariance matrix $P \dot{z}$ and the mutual covariance matrix $P_{X K Z Z}$ :

$$
\begin{gathered}
\chi_{\mathrm{k}, k-1}=H_{k}\left(\xi_{i, k-1}, \mathrm{v}_{k}\right)+\mathrm{r}_{k}, i=0,1, \ldots, 2 \mathrm{n} \\
\hat{Z}_{k, k-1}=\sum_{i=0}^{2 n} \omega_{i}^{(\mathrm{m})} \chi_{i,(\mathrm{k}, \mathrm{k}-1)}=\sum_{i=0}^{2 n} \omega_{i}^{(\mathrm{m})} H_{k}\left(\xi_{i, k-1}, \mathrm{v}_{k}\right)+\mathrm{r}_{k} \\
P_{\overline{\mathrm{Z}} k}=\sum_{i=0}^{2 n} \omega_{i}^{(\mathrm{o})}\left(\chi_{i,(\mathrm{k}, \mathrm{k}-\mathrm{l})}-\hat{Z}_{k, k-1}\right)\left(\chi_{i,(\mathrm{k}, \mathrm{k}-1)}-\hat{Z}_{k, k-1}\right)^{T}+R_{k} \\
P_{X k Z k}=\sum_{i=0}^{2 n} \omega_{i}^{(\mathrm{c})}\left(\xi_{i,(\mathrm{k}, \mathrm{k}-1)}-\hat{X}_{k, k-1}^{a}\right)\left(\chi_{i,(\mathrm{k}, \mathrm{k}-1)}-\hat{Z}_{k, k-1}\right)^{T}
\end{gathered}
$$

So far, we can use the EKF filter algorithm and UKF filter algorithm to extract the line-of-sight angle race of the full-strapdown laser seeker on the rotation missile as the guidance information [12]. It is estimated and simulated as shown in Fig.4 and Fig.5.
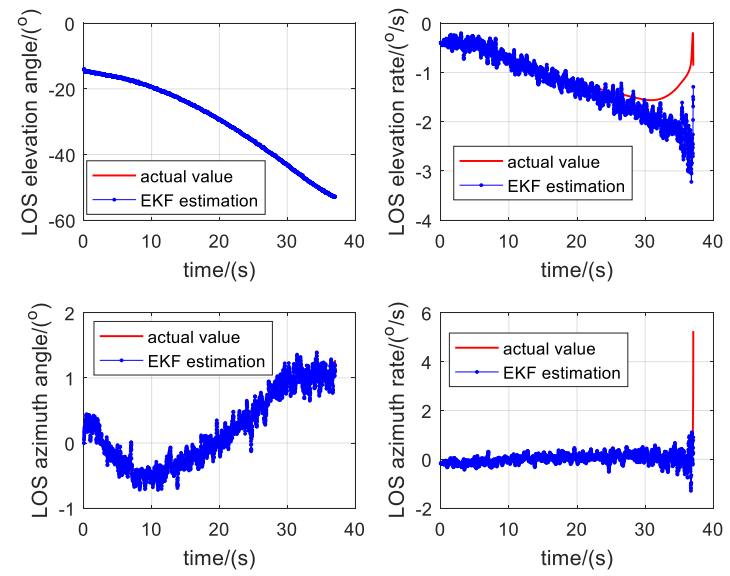

Fig4. EKF filtering line-of-sight and line-of-sight rate estimation.
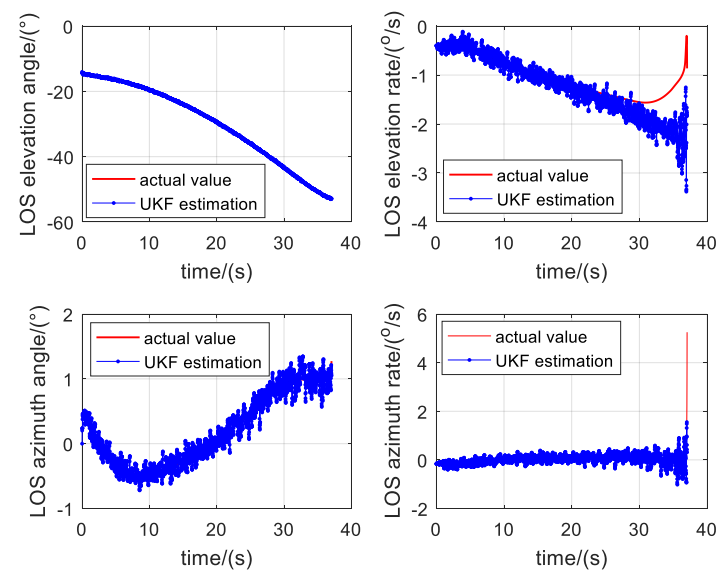

Fig 5. UKF filtering line-of-sight and line-of-sight rate estimation.

\subsection{Computational error analysis}

Calculate the EKF and UKF errors between the filter value and the true value on the line-of-sight elevation angle, the line-of-sight elevation race, the line-of -sight azimuth angle and the line-of-sight azimuth race.
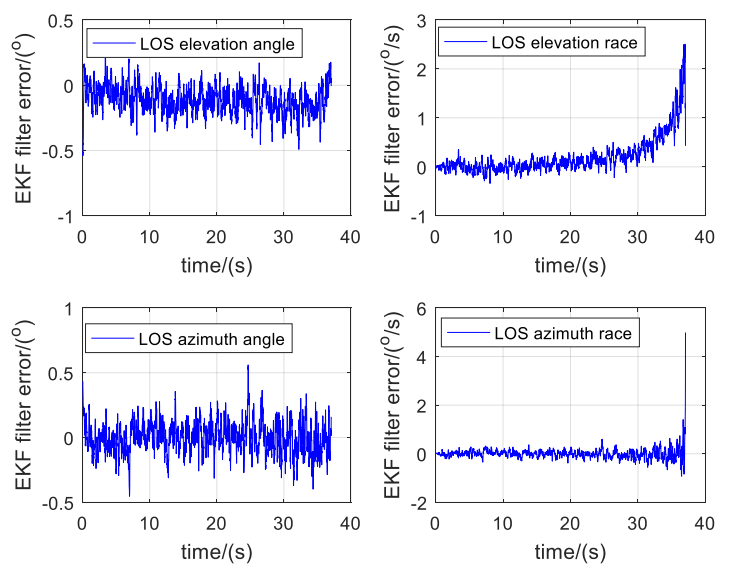

Fig 6. Errors between EKF filter and true value. 

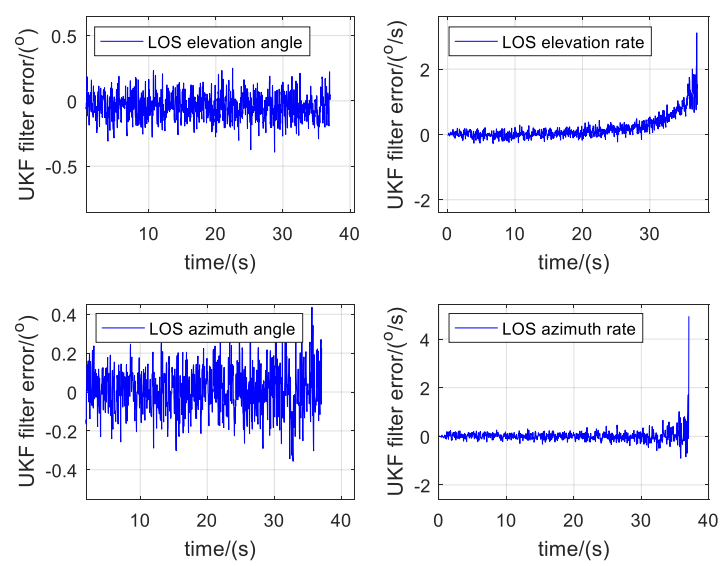

Fig 7. Errors between UKF filter and true value.

It can be seen from the Fig.6 and Fig. 7 that if the nonlinearity is not strong, the results of the EKF filter and the UKF filter are similar. But in the case of high nonlinearity such as the line-of-sight elevation race, the UKF filtering is better than the EKF filtering.

Calculate the EKF and UKF mean error between the filter value and the true value of the on the line-of-sight angle and the line-of-sight rate gets the results shown in Table 1:

Table 1. Mean error of EKF and UKF filtering information.

\begin{tabular}{|c|c|c|}
\hline Mean error & EKF filter & UKF filter \\
\hline LOS elevation angle & 0.234426 & 0.088877 \\
\hline LOS elevation race & 0.347482 & 0.133783 \\
\hline LOS azimuth angle & 0.212946 & 0.092201 \\
\hline LOS azimuth race & 0.318609 & 0.186944 \\
\hline
\end{tabular}

Calculate the root-mean-square error ((RMSE) of the EKF filter and UKF filter can obtain Table.2.

Table 2. RMSE of EKF and UKF filtering information.

\begin{tabular}{|c|l|l|}
\hline RMSE & EKF filter & UKF filter \\
\hline $\begin{array}{c}\text { LOS elevation } \\
\text { angle }\end{array}$ & 0.115062 & 0.095683 \\
\hline $\begin{array}{c}\text { LOS elevation } \\
\text { race }\end{array}$ & 0.354065 & 0.194481 \\
\hline $\begin{array}{c}\text { LOS azimuth } \\
\text { angle }\end{array}$ & 0.104441 & 0.085619 \\
\hline LOS azimuth race & 0.204107 & 0.142643 \\
\hline
\end{tabular}

\section{CONCLUSION}

This paper aims at the engineering application problem of the full-strapdown laser seeker on a rotation missile. First establishing a line-of-sight rate information extraction model. Based on the model, using EKF filter algorithm and UKF filter algorithm to filter and estimate the lineof-sight angle and rate information of the full-strapdown laser seeker. And verifying the filtering algorithm by MATLAB simulation. The results show that UKF filter is better than EKF filter and can be used to extract the guidance information of full-strapdown seeker for rotation missile more accurate.

In this paper, because of time constraints we just targeted research the full-strapdown on rotation missile, other types of seeker algorithms, such as infrared radar, can be further studied. And if more time permits, the control system of rotating projectile can be further studied to realize projectile body integration.

\section{References}

1. Creagh MA, Mee D J. Attitude guidance for spinning vehicles with independent pitch and yaw control $[\mathrm{J}]$. Journal of guidance, control, and dynamics, 33(3),(2010)

2. Ehrich R D, Vergez P. Strapdown seeker technology for the terminal guidance of tactical weapons[C],AGARD Guidance and Control Aspects of Tactical Air launched Missiles 15p,1980, 81(10): 7-15.

3. Kim, D., Ryoo, C. K., Kim, Y., and Kim, J., Guidance and Control for Missile with a Strapdown Seeker, Proceedings of the 11 th International Conference on Control, Automation and Systems, Vol. 86, IEEE Publ.,Piscataway, NJ, 2011, pp. 969-972.

4. Bai Rui,Xia Qunli, Zhang Daochi, Technology of line-of-sight rate estimation using STCKF for strapdown seeker, Infrared and Laser Engineering, Vol.46 No.11,(2017)

5. Sun T, Chu H, Zhang B, et al. Line-of-sight rate estimation based on UKF for strapdown seeker [J].Mathematical Problems in Engineering, 2015, 2015(1): 1-14.

6. Waldmann J.Line-of-sight rate estimation and linearizing control of an imaging seeker in a tactical missile guided by proportional navigation. IEEE Transactions on control systems technology.10, (2002)

7. Li, J. J., "Research of LOS Rate Estimation Method for Strapdown Imaging Seeker," M.S. Thesis, Harbin Inst. of Technology, Harbin, Heilongjiang Province, PRC, (2008).

8. Qian Xingfang, Lin Ruixiong, Zhao Yanan, Missile flight mechanics (in Chinese), Beijing Institute of Technology Press, China, (2013).

9. James, M. M., "Line of Sight Rate Estimation for Guided Projectiles with Strapdown Seeker," AIAA GNCC, (2015).

10. Joongsup Yun, Chang-Kyung Ryoo, Taek-Lyul Song, Strapdown sensors and seeker based guidance filter design, ICCAS, 468-472, (2008).

11. Julier, S. J., and Uhlmann, J. K., "Unscented Filtering and Nonlinear Estimation," Proceedings of the IEEE, No. 3, (2004)

12. Luo Yufeng, Liu Yong, The simulation research on the strapdown inertial navigation algorithm based on the trajectory generator (in Chinese), Journal of Henan Polytechnic University (Natural Science), 06,(2015) 